\title{
Lose Zugehörigkeiten und schwindende Bindungen
}

\author{
Stefan Busse · Karin Lackner ${ }^{1}$ \\ Online publiziert: 30 . Oktober 2018 \\ ๑) Springer Fachmedien Wiesbaden GmbH, ein Teil von Springer Nature 2018, korrigierte Publikation 2018
}

Das immer häufiger wahrgenommene, behauptete oder nur skandalisierte Schwinden von Zugehörigkeit, Loyalität, Anbindung, Verbindlichkeit in lebens- wie arbeitsweltlichen Kontexten scheint eine Chiffre für das spätmoderne Leben zu sein. Langfristiges Gebundensein an Regionen, an Heimat, an Personen, an ,persönliche Objekte“, an Arbeit, an eigene Karriereprojekte, an Klient/innen und Kund/innen, an Gruppen und Teams, an Organisationen und Institutionen etc. schwindet und scheint in den Subjekten einen ambivalenten Widerhall und konkurrierende Resonanzen zu erzeugen.

Auf der einen Seite werden die allgegenwärtigen Flexibilisierungserwartungen und -zumutungen mit einem fluiden Zugehörigkeitsmanagement und mit einer Art flexibilisierenden Bindungsfähigkeit beantwortet, die nicht selten in Attitüden und Inszenierungen zeit- wie raumloser Unverbindlichkeit erstarren. Auf der anderen Seite finden sich Rückzugsbewegungen, die in die Verteidigung mentaler wie räumlicher Grenzsetzungen münden und haltgebende Verbundenheit suggerieren.

Die Lockerung von Einbindungen in zeitliche, räumliche und inhaltliche Rahmungen wird aber auch als ein Zugewinn an Autonomie und Handlungsfreiheit erlebt. Zumindest von jenen, die über die Ressourcen verfügen, diese mit Sinn und Handeln zu füllen. Anderen mag Autonomie als ein schales Versprechen erscheinen, denen Zeitsouveränität und Mobilität als Kompensation für schwindende lebenswie arbeitsweltliche Bindungen ,verkauft“ werden.

Im Grunde genommen haben wir es hier mit dem nicht auflösbaren Widerspruch zwischen Autonomie und Gebundenheit zu tun, der in den letzten Jahrzehnten nur seine spätmoderne Zuspitzung erfahren hat. Gesellschaftliche und politische Institutionen, Organisationen im Profit- wie Non-for-Profit-Bereich, lebens- wie arbeitsweltliche Gruppen und Teams, Familien und Freundschaften, Führung und Leitung können das „Versprechen“, Autonomie zu

\footnotetext{
Dr. Karin Lackner

karin.lackner@aau.at

1 Klagenfurt, Österreich
}

ermöglichen und Gebundenheit zu sichern, immer weniger einlösen. Dennoch sind Zugehörigkeit und Bindung nach wie vor zentrale Werte in ,gemeinschaftlichen Sozialordnungen“ von Betrieben (vgl. Kotthoff 2015, S. 31), Familien, Gemeinwesen etc.

Die gesellschaftspolitischen Veränderungen, der sich zuspitzende Widerspruch zwischen Autonomie und Gebundenheit, zwischen individuellen Interessen und Organisationsinteressen sind auch im organisationalen Alltag angekommen. Neben konkreten, opaken Changeprozessen findet in Organisationen ein fluider Wandel statt, der Organisationen bewegt und die jeweilige Organisation zu entsprechenden Reaktionen herausfordert: „Unternehmen, in denen all dies Realität ist, bieten keine lebenslange Heimat mehr, sondern werden stattdessen zu ,Lebenabschnittspartnern "verbunden mit einem Verlust an Zugehörigkeit". Und für die Subjekte bedeutet das, dass ,die Konstruktionsbedingungen der eigenen Identität wechselnden Anforderungen unterworfene (sind). Sie wird umgeformt und anders ausgerichtet, während man sich durch ständig wechselnde Beziehungen fortbewegt" (Geramanis und Hutmacher 2018, S. XI).

$\mathrm{Zu}$ den Einflussfaktoren fluider Veränderungen zählen:

- Die Digitalisierung der Kommunikation. Computer Mediated Communication eröffnet den Menschen im allgemeinen und Mitarbeiter/innen im besonderen Freiräume, eine Loslösung von Raum- und Zeitstrukturen. Gleichzeitig verdichtet sich ein Angebunden-Sein an das Netz, über welches jeder und jede jederzeit verfügbar ist. Netzwerkkommunikation stellt sowohl eine Art von Nähe her, die fordert - Antworten werden unmittelbar erwartet, als auch eine Distanz - Antworten müssen nicht gegeben werden und Auseinandersetzungen können vermieden werden. Organisationen müssen darüber nachdenken, wie Arbeitsplätze räumlich und zeitlich ausgestaltet werden sollten und welche Spielräume vertragliche Arbeitsvereinbarungen beinhalten könnten, um diesen Widersprüchen gerecht werden zu können.

- Die Lebensentwürfe und Arbeitsplatzvorstellungen der jüngeren Generationen Y und Z. Die heute 20- bis 35- 
Jährigen haben individuelle Vorstellungen darüber, wie sie ihr Leben und ihre Erwerbsarbeit gestalten wollen. Die Verwirklichung der eigenen Lebensentwürfe steht im Vordergrund, vertragliche oder gar emotionale Bindungen an Arbeitgeber/innen werden hintan gestellt. Individualisierung, Selbstoptimierung, Selbstdarstellung, eine ausgewogene Work-Life-Balance, unmittelbare Rückmeldungen $\mathrm{zu}$ ihren Leistungen (instant gratification) sind nur eine kleine Auswahl von Zuschreibungen, verändern jedoch das emotionale Binnenklima in Organisationen und Teams.

- Die Interkulturalisierung in und von Organisationen. Einerseits haben Personen der jüngeren Generation schon während ihrer Ausbildungszeit Gelegenheiten Erfahrungen in und mit anderen Kulturen zu machen. Die Entwicklungen unseres Bildungswesens machen dies möglich. Andererseits wird das Leben mit anderen Kulturen vor Ort beispielsweise durch Migrationsbewegungen erlebbar. Mancherorts entwickelt sich eine Hyper Culture, also ein akzeptiertes Miteinander unterschiedlicher Herkunftskulturen, ohne den eigenen Kulturhintergrund verleugnen zu müssen. Gleichzeitig entstehen „Global Identities“, die sich in unterschiedlichen Kulturen bewegen und ein Metakultur Bewusstsein verinnerlicht haben.

- Die Ausdifferenzierung von Organisationsstrukturen. Organisationen reagieren auf externe und interne Herausforderungen mit strukturellen Ausdifferenzierungen. Das starre hierarchische Grundgerüst von Organisationen wird ergänzt durch diverse andere Systemelemente. Organisationen experimentieren mit alternativen Vernetzungsentwürfen wie agilen Strukturen, Idea Labs, Scrum Teams, u.v.a.m.

- Die Beschleunigung von Prozessen. Organisationen bewegen sich in dem Spannungsfeld von Innovationshektik und Implementierungsroutine. Einerseits verursachen die schon genannten fluiden Veränderungen Handlungsdruck, andererseits sind es die Märkte, Konkurrenten, die Organisationen von einem Veränderungsprozess in den nächsten antreiben.

Wie zeigen sich diese Widersprüche in der gelebten Organisationspraxis? Welche Phänomene können dabei beobachtet werden?

- Organisationen sind, vor allem in technischen Bereichen, mit hoher Mitarbeiter/innen Fluktuation konfrontiert. Mitarbeiter/innen nehmen die Verantwortung über ihre eigenen Karrieren in ihre Zuständigkeit. Die erlebte Freiheit hat ihren Preis: Die für die eigene Karriereplanung notwendige Leistungskontrolle obliegt der individuellen Gewissensinstanz.

- Teams werden Mittel zum Zweck: Erfolgreiche Teamperformance erhöht die Chancen auf einem dynamischen
Arbeitsmarkt. Bindungen in Teams sind zweckorientiert, emotionale Zugehörigkeitsgefühle werden zurückgehalten.

- Klassische T-Gruppen verlieren die Faszination eines sich entwickelnden Gruppengefühls. Individuelle Interessen stellen sich zunehmend vor das Gruppenerleben (,was kann ich aus dem gruppendynamischen Training für mich mitnehmen?").

- Agile Organisationsformen und -strukturen erhöhen die Mehrfachzugehörigkeit von Mitarbeiter/innen. Individuen fühlen sich nirgendwo eindeutig zugehörig, sie werden auf sich selbst zurückgeworfen.

- Globale Organisationen erwarten von ihren Mitarbeiter/innen Auslands-Entsendungs-Bereitschaft. Kulturelle Grenzen werden innerhalb der Organisation ,,aufgehoben“" in dreifacher Wortbedeutung: bewahrt, aufgelöst, auf eine höhere Ebene gehoben. Neue Identitäten werden geschaffen, sogenannte „Glopats“. Kulturelle Unterschiede und daraus resultierende Konflikte werden individualisiert und der jeweiligen Persönlichkeit zugeschrieben.

- Zugehörigkeiten und Bindungen werden gesucht und gefunden in Sozialen Netzwerken. Personen fühlen sich freiwillig gezwungen dabei zu sein. Bei Verweigerung der Teilnahme droht soziale Isolation. Die Zugehörigkeit zu sozialen Netzwerken erzeugt unverbindliche Bindungen einerseits und ein sich dem sozialen Druck ausgeliefert Fühlen andererseits. Zwischen der eigenen Person und den anderen steht das Medium des Internets, Face to Face Kommunikation wird unterbunden. Soziale Kontakte spielen sich in zwei Parallelwelten ab: Im Hier und Jetzt der unmittelbaren Begegnung mit den jeweils anderen und im Dort und Jetzt in den sozialen Netzwerken. Netzwerke ermöglichen Zugehörigkeiten unabhängig von Raum und Zeit. In der direkten Face to Face Kommunikation sind Raum und Zeit (wie beispielsweise in T-Gruppen) rahmengebende konkrete Grenzziehungen und verlangen Verbindlichkeit. Das Netz suggeriert Unverbindlichkeit und Unendlichkeit.

Die Beiträge in diesem Heft beschäftigen sich mit dem Thema Zugehörigkeit und Bindung aus unterschiedlichen Perspektiven.

Die Beziehung von Mitarbeiter/innen und Arbeitgeber/ innen im organisationalen Kontext steht im Fokus der Beiträge von Busse, Schunter, Schmidt und Becke.

Stefan Busse verschränkt in seinem Beitrag „Bindung(en) in der Arbeitswelt" erstmalig die Theorien der Mitarbeiterbindung und der Commitmentforschung mit der Bindungstheorie aus der Entwicklungspsychologie und kommt zu erstaunlichen Übereinstimmungen. Menschliche Arbeit schafft bindungsrelevante Beziehungen; Bindungsmuster regulieren das Verhalten zur Organisation und $\mathrm{zu}$ 
Personen; Bindungsmuster und bindungsrelevantes Verhalten sind veränderbar; in der Interaktion treffen unterschiedliche Bindungsmuster aufeinander und beeinflussen sich wechselseitig. Die bindungstheoretische Reflexion von Arbeitsbeziehungen resp. Beziehungsarbeit öffnet den Blick auf Beziehungsmuster. Führungskräfte, Berater/innen, Coaches sollten eine Sensibilisierung für Bindungsmuster entwickeln, um sich in agilen und von Individualisierung durchwirkten Arbeitssituationen professionell bewegen zu können.

Angelika Schmidt zeigt in ihrem Beitrag ,Verwerfungslinien in Organisationen - die Wirkung von Kontextfaktoren auf die Verbundenheit in Organisationen“, wie sich in Organisationen resp. Unternehmen makrostrukturelle Flexibilisierungsanforderungen und -zumutungen (des Arbeitsmarktes) über die mesostrukturellen Steuerungs- und Kontrollmöglichkeiten der Beschäftigungspolitiken bis zur mikrostrukturellen Ebene von Interaktion und Beziehung zwischen den Beschäftigten und Beschäftigern fortsetzen. Dabei steht zum einen die Frage im Mittelpunkt, wie Unternehmen aus den Flexibilisierungsmöglichkeiten und damit dem verändertem organisationalen Commitment der Beschäftigen Wettbewerbsvorteile zu ziehen vermögen. Zum anderen wird gezeigt, welche Risiken daraus erwachsen können, weil sich aus den unterschiedlichen Dispositionsmöglichkeiten hinsichtlich Zeit, individueller Handlungsspielräume und erlebter sozialer Gerechtigkeit Schräglagen entwickeln und latente Verwerfungslinien (faultlines) entstehen und „zum Kochen gebracht“ werden können.

Guido Becke nimmt in seinem Beitrag „Fragmentierung sozialer Zugehörigkeit in Unternehmen durch Ökonomisierung" diesen Faden in gewissem Sinne auf und zeigt anhand von zwei Betriebsfallstudien, wie die Ökonomisierungsstrategien auf Organisationsebene zu ungeplanten Fragmentierungsprozessen auf Teamebene führen. Hier werden Etablierten-Außenseiter- resp. Kern- und Randgruppen-Dynamiken in Gang gesetzt die interaktiv als unterschiedlich starke soziale Zugehörigkeit resp. Bindungen agiert werden. Die Rolle von Führung als wichtiger Mediator in der reflexiven Bearbeitung von solchen Fragmentierungen sozialer Zugehörigkeit wird herausgestellt.

Jürgen Schunter versucht in seinem Beitrag „Mitarbeiterintegration und Mitarbeiterbindung von Organisationen - Wie integrieren und binden Organisationen ihr Personal?" auf einem dezidiert systemischen Theorietableau zunächst das Thema Zugehörigkeit als Kopplungsphänomen und -problem zwischen System (Organisation) und Umwelt (Mitarbeiterrollen) zu skizzieren. Dabei identifiziert er auf dem Hintergrund einer empirischen Untersuchung an Weiterbildungsorganisationen einen ,semantischen Integrationsmechanismus", der wie eine Membran funktioniert und über diverse semantische Angebote kommunikative Bindung und Integration der Mitarbeitenden an die Organisa- tion erzeugt und erzeugen soll. Dabei werden empirisch eine Reihe „habitueller Typen“ unterschieden, die über verschiedene Codes „Spezialsemantiken“ herausbilden, die sozusagen als latente Folien der Kommunikation Bindung des Personals einer Organisation ermöglichen aber auch verhindern können.

Die Beiträge von Lackner, Angeli und Andrione betrachten das Thema Zugehörigkeit und Bindung aus einer Generationenperspektive.

Der Beitrag von Ludwig Andrione „Eigenschaften, Einstellungen und Werte von Generationen: Stand und Aussicht der Forschung" gibt einen kurzen historischen Überblick über die Themen und Ergebnisse der Generationenforschung.

Michael Angeli widmet sich in seinem Beitrag „Generationen-Management und Mitarbeiterbindung. Effekte und Maßnahmen“ dem Thema Generationenmanagement. Immer häufiger sind Führungskräfte mit sogenannten „,mixed generation teams “ und den damit verbundenen Interessensund Einstellungsunterschieden unterschiedlicher Generationen befasst. Angeli präsentiert Auszüge aus einem empirischen Forschungsprojekt, durchgeführt in verschiedenen Wirtschaftsorganisationen. Welche Faktoren zu einer besseren Mitarbeiter/innen-Bindung wirksam sein könnten, wird in einer Interkorrelationsmatrix dargestellt und Maßnahmen für Personalentwicklung und Management abgeleitet. Um als Unternehmen für jüngere Generationen attraktiv zu sein, braucht es aktive Schaffung von Karrieremöglichkeiten und gute Führung. In dem Beitrag wird ausführlich dargestellt, was jeweils darunter zu verstehen ist.

Der Beitrag von Karin Lackner „Millennials und Nexters. Veränderungen von Interaktions-, Beziehungs-, Bindungs- und Affektmuster in gruppendynamischen Trainingsgruppen“ geleitet die Leser/innen durch ein gruppendynamisches Forschungsprojekt, durchgeführt an der Universität Klagenfurt im Rahmen eines Forschungspraktikums. Wie zeigt sich der Generationenunterschied in einer klassischen Trainings Gruppe? Beobachtungen von langjährigen Trainer/innen, dass sich die Zugehörigkeitsdichte in T-Gruppen zu Gunsten einer zunehmenden Individualisierung verringert, waren Impulse für das Projekt, das aus vier Teilschritten bestand. Hauptteil der Forschung war eine teilnehmende externe Beobachtung von T-Gruppen über einen Zeitraum von einer Woche und eine Befindlichkeitsabfrage. Die Ergebnisse bestätigen die Eingangsvermutung und liefern darüber hinaus interessante Erkenntnisse über die Generationen Y und Z.

Das Thema Zugehörigkeit und Bindung, betrachtet aus einer kulturellen Perspektive, greift Ariane-Sissy Wagner in ihrem Beitrag „Lose Zugehörigkeiten von globalen Identitäten (Glopats) in internationalen Unternehmen - Konflikte zwischen Nationalkulturen und Hyperkultur" auf. Auch dieser Beitrag ist ein Auszug aus einem größeren Forschungs- 
projekt zum Thema interkulturelle Konflikte. Der Beitrag eröffnet eine Welt der Vielfalt und Komplexität von globalen Organisationswelten. Deutlich wird dabei der Widerspruch von kultureller Identität und Zugehörigkeit und dem Verlust derselben bei globalen Identitäten bzw. in einem Hyper Culture Milieu. Die Schlussfolgerungen von Wagner liefern Impulse für Personalentwicklung und Management von internationalen Organisationen.

Geht es in den bisher beschriebenen Beiträgen um schwächer werdende Bindungen, lose Zugehörigkeiten und die Bemühungen und Strategien von Organisationen mit diesen umzugehen, so beschreibt der Beitrag von Ruth Lerchster „Unternehmensnachfolge als ,Rites de passage“. Zugehörigkeit, familiäre Loyalität, Tradition, Beziehung und emotionale Gemengelage als machtvolle Einflussgrößen in Übergabeprozessen“ das genaue Gegenteil. Hier geht es um das Dilemma von unauflösbaren familiären Bindungen in Familienunternehmen und dem Druck der Traditionen. Betrachtet man die aktuellen fluiden Veränderungen in Organisationen, so verschärft sich in Familienunternehmen der Widerspruch von Autonomie und Gebundenheit. Der Text entführt die Leser/innen in das Treibhaus von Gefühlslagen und Widersprüchen bei Übergabeprozessen und verdeutlicht die systemdynamischen Spannungsfelder aus einer sozial analytischen Perspektive.

\section{Literatur}

Geramanis, O., \& Hutmacher, St (2018). Vorwort: Identität in der modernen Arbeitswelt - etwas Seiendes oder Werdendes. In O. Geramanis \& S. Hutmacher (Hrsg.), Identität in der modernen Arbeitswelt. Neue Konzepte für Zugehörigkeit, Zusammenarbeit und Führung (S. IX-XIV). Wiesbaden: Springer Gabler.
Kotthoff, H. (2015). Zugehörigkeit - womit und wofür? Betriebssoziologische, betriebswirtschaftliche und organisationspsychologische Perspektiven. Supervision. Mensch, Arbeit, Organisation. Die Zeitschrift Für Beraterinnen und Berater, 33(2), 28-37.

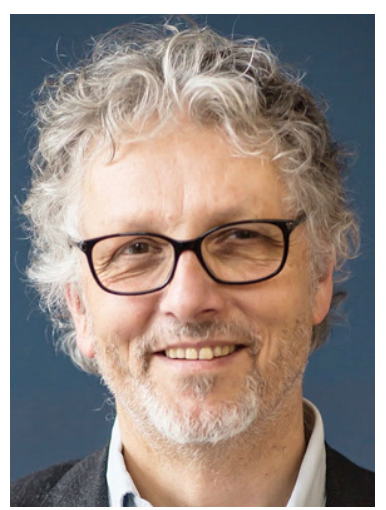

Prof. Dr. rer. nat. habil. Dipl. Psychologe Stefan Busse Fakultät Soziale Arbeit der Hochschule Mittweida, Studiengangsleiter der Zertifikatsstudienganges ,Supervision und Coaching“ und „Training für Kommunikation und Lernen in Gruppen“" an der Hochschule Mittweida, Direktor des Institutes für „Kompetenz, Kommunikation und Sport“ (IKKS), Ausbildungsleitung bei Basta Fortbildungsinstitut für Supervision und Coaching e. V.

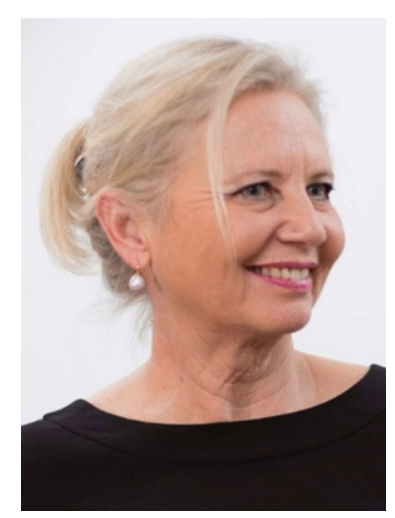

Dr. Karin Lackner Universität Klagenfurt. Bis 12/2016 Professorin an der Universität Kassel für Organisationsberatung, Supervision und Coaching. Lehrberaterin und Lehrtrainerin (ÖGGO), Mitglied der DGSv, eingetragene Gesundheitspsychologin in Österreich. Gründung und Leitung des Instituts für Organisationsdynamik. Beratungs-, Trainingsund Forschungstätigkeit im In- und Ausland. 